\section{Grippeschutz für Senioren mit adjuvierter Vakzine}

Bei älteren Menschen führt die Influenza häufig zu schweren Komplikationen, die tödlich verlaufen können. Mit einer adjuvierten Influenza-Vakzine kann in dieser Altersgruppe ein besserer Grippeschutz erzielt werden als mit einem konventionellen Grippeimpfstoff.

- Eine Influenza-Impfung mit konventionellen Vakzinen bietet nur einen begrenzten Grippeschutz, da die Immunantwort mit zunehmendem Alter schwächer ausfällt. Daher benötigen Senioren angesichts ihres Immunstatus und des damit verbundenen Risikos für schwere Krankheitsverläufe einen besonderen Grippeschutz.

Diese Anforderung erfüllt die auf die immunologischen Besonderheiten dieser Altersgruppe zugeschnittene Influenza-Vakzine Fluad $^{\oplus}$ : Anders als konventionelle Grippeimpfstoffe ruft sie aufgrund des Wirkverstärkers MF59 auch bei über 65-Jährigen eine gute Immunantwort hervor. Besonders ausgeprägt ist die stärkere Antikörperbildung gegen Influenza-Antigene des
Typs A/H3N2. Diese Antigene verursachen bei älteren Menschen die häufig schweren Krankheitsverläufe und die damit verbundenen Komplikationen. 80\% der Grippeassoziierten Todesfälle sind auf die A/H3N2- Antigene zurückzuführen [1].

\section{Risiko für Klinikeinweisungen deutlich gesenkt}

Die klinische Effizienz des wirkverstärkten Grippeimpfstoffs wurde in zwei groß angelegten Studien nachgewiesen. Bei der ersten Arbeit handelte es sich um eine prospektive Beobachtungsstudie mit mehr als 107.000 Patienten im Alter über 65 Jahren, die über drei konsekutive Grippesaisons (2006-2009) in Norditalien lief. Sie ergab,

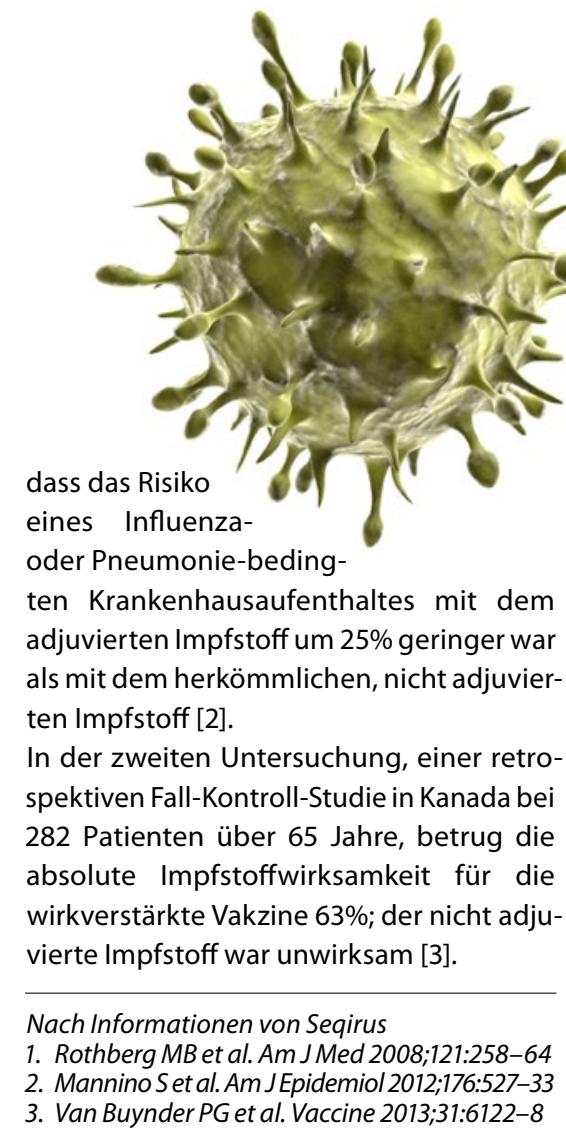

\title{
FORTA-Liste empfiehlt Gliptine
}

Gerade für die antidiabetische Therapie älterer Patienten gilt: Sicherheit geht vor Wirksamkeit. Diesem Anspruch werden Gliptine in besonderem Maße gerecht, sodass diese Substanzgruppe in der FORTA-Liste als einziges orales Antidiabetikum auch in Kombination mit Metformin empfohlen wird.

— Ältere Menschen sind in der Regel polymorbide. Daraus resultiert häufig eine Polymedikation, die wegen potenzieller Interaktionen Risiken birgt. Eine verminderte renale und hepatische Funktion beeinflusst den Abbau vieler Medikamente, dadurch besteht zusätzlich die Gefahr der Kumulation. „Deshalb treten häufiger schwere Nebenwirkungen auf", erklärte Prof. Martin Wehling, Direktor des pharmakologischen Instituts der Universität Mannheim.

\section{Sichere Substanzen auswählen}

Besonders gefürchtet sind medikamentös induzierte Verwirrtheitszustände, wie sie auch durch eine antidiabetische Therapie - genauer gesagt durch Hypoglykämien verursacht werden können. Deshalb ist es wichtig, gerade bei älteren Patienten sorgfältig Substanzgruppen auszuwählen, die ein Höchstmaß an Sicherheit bieten.
"Im Alter geht Sicherheit vor Wirksamkeit", sagte Wehling.

\section{Welche Medikamente sind geeignet?}

Um die Sicherheit der medikamentösen Therapie zu verbessern, wurde die FORTA(Fit fOR The Aged)-Liste erarbeitet. Damit werden die Medikamente dahingehend bewertet, ob sie für alte Patienten geeignet sind. „Wir konnten im Rahmen einer Studie zeigen, dass bei Beachtung dieser Empfehlungen signifikant weniger Nebenwirkungen auftreten", berichtete Wehling.

Gliptine wie Sitagliptin (Xelevia ${ }^{\circledast}$ ) erhielten als einzige Antidiabetika die Bewertung A, sie sind also besonders für ältere Diabetiker geeignet, evtl. auch in Kombination mit Metformin, vor allem wegen des fehlenden Hypoglykämierisikos. Sulfonylharnstoffe werden dagegen der Gruppe D zugeordnet. Dies entspricht der Empfehlung "nicht ein- setzen bzw. absetzen“. Gleiches gilt auch für die SGLT2-Inhibitoren, weil unter diesen Substanzen häufiger urogenitale Infektionen auftreten. „Und diese verlaufen bei älteren Menschen nicht selten septisch und auch tödlich", warnte Wehling.

Dr. med. Peter Stiefelhagen

Satellitensymposium „Die Vielfalt des Typ-2Diabetes - Ein Blick ins Wartezimmer", Fortbildungsveranstaltung "Innere Medizin fachübergreifend - Diabetologie grenzenlos", Unterschleißheim, Juli 2017, Veranstalter: Berlin-Chemie

SilverStar-Preis 2018 fördert Hilfe älterer Menschen mit Diabetes

Mit dem Föderpreis "SilverStar” zeichnet das Unternehmen Berlin-Chemie Projekte für die Versorgung von älteren Diabetikern aus. Der Preis ist mit insgesamt $25.000 €$ dotiert. Egal ob privates, ehrenamtliches oder berufliches Engagement: Wichtig ist, dass das Projekt die Lebensqualität älterer Menschen mit Diabetes nachhaltig verbessert. Unter www.silverstar-preis.de können Sie sich jetzt bewerben. Die Bewerbungsfrist endet am 31. Dezember 2017.

Nach Informationen von Berlin Chemie 